\title{
Recherches dans la province du Jiangsu (Chine)
}

Research in Jiangsu province, China

\section{Antoinet Schimmelpenninck}

Traducteur : Ramèche Goharian

\section{OpenEdition}

\section{Journals}

Édition électronique

URL : http://journals.openedition.org/ethnomusicologie/1187

ISSN : 2235-7688

\section{Éditeur}

ADEM - Ateliers d'ethnomusicologie

\section{Édition imprimée}

Date de publication : 31 décembre 1995

Pagination : 33-57

ISBN : 2-8257-0537-3

ISSN : $1662-372 X$

\section{Référence électronique}

Antoinet Schimmelpenninck, «Recherches dans la province du Jiangsu (Chine) », Cahiers

d'ethnomusicologie [En ligne], 8 | 1995, mis en ligne le 04 janvier 2012, consulté le 19 avril 2019. URL :

http://journals.openedition.org/ethnomusicologie/1187

Ce document a été généré automatiquement le 19 avril 2019

Tous droits réservés 


\title{
Recherches dans la province du Jiangsu (Chine) ${ }^{1}$
}

Research in Jiangsu province, China

\author{
Antoinet Schimmelpenninck
}

Traduction : Ramèche Goharian

\section{La région de $\mathrm{Wu}$}

1 De 1987 à 1992, j'ai fait mes recherches dans le Jiangnan, situé dans le Jiangsu Sud, où prédomine le dialecte $\mathrm{Wu}$, en réalité un groupe de dialectes apparentés ${ }^{2}$. J'utiliserai ici le terme « région de $\mathrm{Wu}$ » pour parler de cette partie limitée, située à l'est et au sud-est du lac Tai où j'ai mené l'essentiel de mon enquête (fig. 1, p. 34). La véritable région de Wu, c'est-à-dire la sphère d'influence réelle des dialectes $\mathrm{Wu}$, s'étend bien plus loin, même si ses frontières exactes restent à discuter (fig. 2, p. 35). Le district de Nankin, qui se trouve au sud du Jiangsu, n'appartient pas à cette grande région de $\mathrm{Wu}$, mais le district de Shanghai en fait partie. Certaines régions situées sur la rive nord du Yangzi, ainsi que la plus grande partie de la proviynce de Zhejiang, y sont incluses.

2 J'ai concentré l'essentiel de mes recherches sur la région qui borde le lac Tai à l'est et au sud-est et dont le centre est la ville de Suzhou (env. 750000 habitants). Autrefois, cette région se trouvait au cœur du royaume semi-barbare de $\mathrm{Wu}\left(\mathrm{V}^{\mathrm{e}}\right.$ siècle av. J.-C.) ; mais plus tard, différents royaumes ou états vassaux et de nombreux districts et villes de cette région adoptèrent le nom de Wu. D’où le terme «Wu ge ", employé dans les documents historiques et littéraires, pour désigner les chants populaires de cette (plus large) région de $\mathrm{Wu}^{3}$.

3 Ayant entièrement concentré mon enquête sur la campagne, je n'ai pas essayé de recueillir les chants populaires à Suzhou ou dans d'autres centres urbains. Les chants collectés pour cette étude proviennent de plus de cinquante villages situés dans les districts de $\mathrm{Wu}$ (Wuxian), Wujiang, Wuxi, Changshu, Taicang, Kunshan et Jiashan (province du Zhejiang). A cela s'ajoute l'île de Chongming, le tout représentant une 
superficie totale d'environ $8000 \mathrm{~km}^{2}$. Actuellement, la population rurale de cette région s'élève à près de 5 millions d'habitants ${ }^{4}$.

Fig. 1 : Carte de la région où j'ai effectué la plus grande partie de mes recherches sur le terrain.

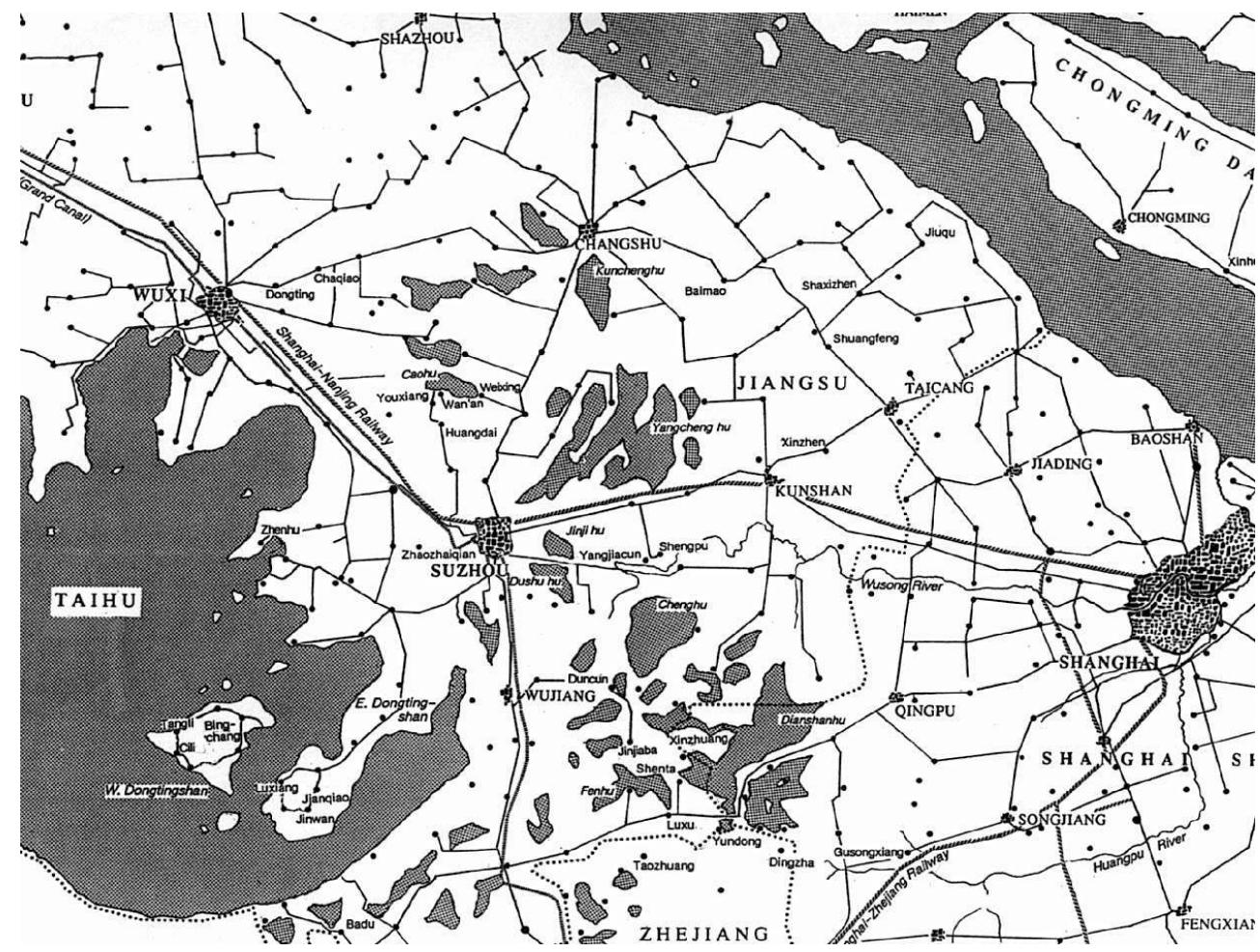

Fig. 2 : La région dialectale de Wu est située grosso modo à l'intérieur de la ligne pointillée.

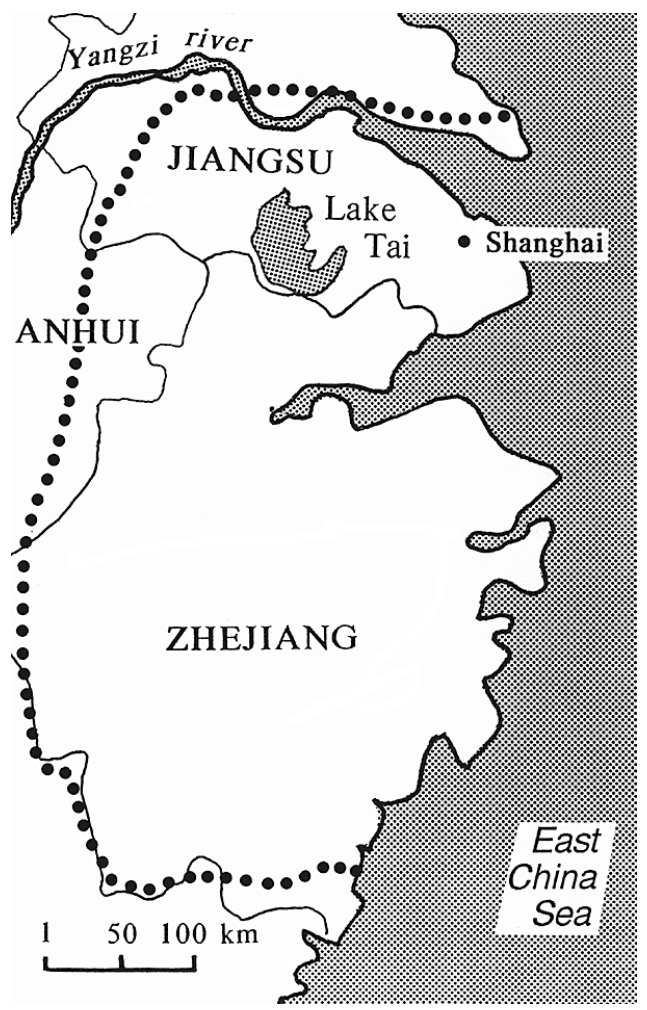




\section{La tradition du chant populaire dans la région de $\mathrm{Wu}$}

Dans l'ensemble, les jeunes ne s'intéressent plus aux chants populaires. Mais qu'en est-il de ceux pour qui ces chants revêtent encore, ou revêtaient à un certain moment, une grande importance? Ceux qui tombaient amoureux lorsqu'ils chantaient avec des compagnons et qui avouaient leur amour dans les chansons populaires? Ceux qui supportaient les heures interminables d'un travail agricole éreintant grâce au chant qui les aidait à oublier tout le reste? Ou encore ceux qui chantaient, pendant les longues soirées d'été, l'histoire des guerriers héroïques, des amants malheureux et des événements miraculeux, sur le pas de leur porte?

5 Dans la région de $\mathrm{Wu}$, il reste encore quelques chanteurs populaires, même jeunes, mais leur nombre décroît. J'ai fondé mon étude sur des matériaux collectés auprès de cent chanteurs que j'ai découvert avec l'aide des fonctionnaires locaux. De mai 1987 à avril 1992, j'ai mené environ quatre-vingts entretiens avec ces chanteurs et avec des chercheurs locaux de chants populaires. J'ai aussi discuté avec des musicologues à Pékin, à Shanghai et à Nankin. Grâce à ces entretiens et aux séances de chant, j'ai pu me faire une idée d'ensemble de la tradition du chant populaire $\mathrm{Wu}$.

6 Le plus jeune chanteur que j'ai rencontré avait 12 ans en 1992, et le plus vieux en avait 90, mais en général tous ceux que j'ai enregistrés pour cette étude étaient âgés de 60 à 80 ans. Sans aucun doute, la tradition du chant a commencé à décliner dans la région de Wu dès le début des années 1950 et elle disparaitra vraisemblablement dans les prochaines décennies. A l'exception des régions de Baimao et de Jiashan où l'on continue de transmettre les chants aux jeunes générations, il ne reste plus qu'une poignée de personnes capables d'exécuter ces chants populaires dans les autres villages que j'ai visités. Environ deux tiers de ces chanteurs étaient des hommes. Ce qui m'a frappé plus encore que l'âge avancé des chanteurs était l'absence totale d'un public dans les villages pour les écouter. Mon enquête a certes suscité quelque intérêt auprès du public, mais les chanteurs n'auraient jamais chanté si moi-même ou d'autres collecteurs de chants populaires n'étions venus le leur demander.

7 Tous les chanteurs que j'ai interrogés me l'ont confirmé : jusque dans les années 1950, les chants populaires faisaient normalement partie de la vie campagnarde et les jeunes participaient activement au maintien de cette tradition. La pratique de shan'ge notamment était liée aux travaux des champs et constituait la distraction des soirées d'été de la population lorsque les villageois se réunissaient devant la maison d'un chanteur local pour écouter, dans la fraîcheur de la brise, ses chansons et ses histoires. Mais les chants ne sont plus du tout exécutés dans ce contexte: de nos jours, on les chante à certaines occasions seulement, comme pendant les séances d'enregistrements ou lors de l'assemblée annuelle de la Société pour la recherche sur le chant populaire $\mathrm{Wu}$, fondée en 1985. A cet égard, la région de Wu contraste de façon frappante avec la région voisine de Nantong, dans le Nord, par exemple. Là, les chants populaires ont aussi beaucoup perdu de leur fonction initiale, mais on peut encore parfois les entendre, accompagnant les travaux des champs; et dans les villages de trente ou quarante habitants de cette région, on trouve encore des chanteurs compétents parmi la population jeune ou plus âgée. La pénurie actuelle de chanteurs dans la région de $\mathrm{Wu}$ peut être imputée à l'industrialisation rapide de cette région et à la proximité de Shanghai, un des lieux d'origine de la Révolution Culturelle. 
8 Pendant la Révolution Culturelle (1966-1976), il était interdit de chanter des chansons populaires; les gardes rouges persécutaient et maltraitaient des chanteurs pleins de talent et ils brûlèrent de grandes quantités de recueils de chants et d'autres documents écrits. J'ai remarqué que, dans la région de $\mathrm{Wu}$, beaucoup de gens avaient encore peur à cause de leurs expériences passées et ne voulaient plus chanter, surtout devant un microphone. Les collecteurs amateurs de la Société pour la recherche sur le chant populaire $\mathrm{Wu}^{5}$, qui avaient commencé à visiter les villageois dès 1979, avaient parfois mis deux à trois ans pour convaincre les chanteurs que leur art n'était plus considéré comme une activité dangereuse ou contre-révolutionnaire et que le gouvernement encourageait même la recherche scientifique et la conservation des chants populaires, y compris les chansons d'amour.

comment pourra-t-on jamais combler le vide laissé par tant d'années de silence imposé ? La jeune génération a perdu tout lien avec la tradition ancienne et son intérêt musical se porte sur le répertoire populaire de la radio (essentiellement la musique pop). Le gouvernement s'intéresse de moins en moins aux chansons populaires comme outil de propagande. Les grandes manifestations comme le festival de chant qui se tenait chaque année à la mi-automne sur la rivière Baimaotang, attirant des milliers de spectateurs, entre 1949 et 1972, n'ont plus lieu.

10 Dans la région de $\mathrm{Wu}$, les quelques membres de la jeune génération qui s'intéressent encore aux chants populaires les étudient auprès de leurs parents ou des anciens de leurs villages; mais le processus d'apprentissage a fini par ressembler à celui des écoliers qui apprennent à chanter. La transmission ne se fait plus de manière spontanée ; les jeunes transcrivent les textes et veulent avoir une version écrite à disposition lorsqu'ils chantent. La plupart d'entre eux n'aiment d'ailleurs pas chanter des chansons à moins d'y être spécialement invités, comme par exemple pendant les festivals de chants organisés par les bureaux de la culture locaux. A ces occasions, ils montent sur scène et chantent avec un microphone, parfois accompagnés d'instruments chinois ou occidentaux (alors que ces chants n'ont jamais été censés être interprétés de cette façon).

11 Une poignée de jeunes témoignent d'un intérêt professionnel pour le chant ; ils étudient dans les conservatoires et dans les écoles d'art où l'on enseigne un chant cultivé dans le style de l'opéra occidental (souvent avec accompagnement d'instruments modernes), bien éloigné du style rural traditionnel.

12 On peut deviner à quelle vitesse la population de la région de Wu a changé d'attitude à l'égard du chant populaire en entendant cette réponse des vieux chanteurs lorsqu'on leur demande pourquoi ils ne chantent plus dans les champs : «Nous ne pouvons pas le faire. Les gens se moqueraient de nous; ils nous prendraient pour des fous!» Les chanteurs que j'ai enregistrés appartiennent au noyau de ce qui fut jadis une riche tradition vocale à laquelle beaucoup de monde participait jusque dans les années 1950.

13 Mais quel était exactement leur nombre dans le passé ? Combien de personnes chantaient effectivement en ces temps-là? Il est difficile de répondre à ces questions. Lorsqu'ils parlent de leur enfance, les chanteurs répètent invariablement: "tout le monde chantait ». Je présume qu'un grand nombre de gens connaissaient ces chants même si la plupart d'entre eux n'en avaient qu'une connaissance partielle. Il est certain que tous ne chantaient pas ; je ne pense d'ailleurs même pas que ceux qui chantaient se considéraient nécessairement comme des "chanteurs». Les chanteurs, quels qu'ils fussent, ne constituaient certainement pas un groupe homogène d'un point de vue culturel ou social. 
$\mathrm{Au}$ sein de leur communauté, une élite se considérait (et était acceptée) comme l'interprète clef $\mathrm{du}$ répertoire. Ces hommes et ces femmes n'appartenaient pas nécessairement à une seule couche sociale, mais le contenu de certains poèmes laisse supposer que, dans un passé reculé, les chanteurs principaux exerçaient la fonction de prêtres et de chamanes dans leur communauté, dans les villages de la région de $\mathrm{Wu}^{6}$.

\section{Le déclin des chants populaires Wu} déclin rapide de la culture du chant populaire $W u$ au $x^{e}$ siècle. J'ai déjà mentionné les effets désastreux de la Révolution Culturelle. En fait, de nombreux chanteurs m'ont avoué qu'ils n'avaient pas chanté pendant près de quarante ans parce qu'ils avaient refusé de participer aux campagnes de chansons organisées par la propagande communiste au début des années 1950. A cette époque, on dissuadait déjà les villageois de chanter les textes traditionnels qui contenaient, prétendait-on, des « éléments superstitieux » et les «vestiges de la mentalité féodale esclavagiste». Par contre, on les encourageait à composer sur les airs anciens de nouvelles paroles, de nouveaux textes: messages patriotiques, réflexions réalistes sur le progrès sous le régime communiste, louanges du parti, etc. De nos jours, on peut encore entendre certains de ces chants dans la région de Wu. Cette évolution n'enchantait évidemment pas tout le monde. Mais, qu'ils fussent enthousiastes ou non, les chanteurs populaires ne pouvaient pas échapper à l'impact de cette période. Ses conséquences ultimes sur la culture locale sont illustrées par ces paroles d'un chanteur, Zhao Yongming :

" Je ne savais pas lire, la vie était dure à la maison, nous étions très pauvres. Nous n'avions pas d'argent pour aller à l'école. Mais depuis que le parti communiste a fondé la Nouvelle société, les enfants du peuple ont des livres pour lire. Ils forment une nouvelle et belle génération qui peut lire et travailler. [...] Sous le Guomindang, les enfants de familles pauvres n'avaient pas de livres pour lire, ils ne pouvaient que garder les vaches. Ah, c'était dur, on ne pouvait pas lire, pas un seul caractère !»

Apparemment, les slogans politiques préfabriqués, contenus dans les textes des «nouveaux chants populaires ", étaient non seulement incorporés dans les anthologies et adaptés aux chants locaux, mais ils s'étaient en plus infiltrés dans le vocabulaire et le langage quotidiens de certains chanteurs. Pourtant la région de Wu n'était pas aussi « analphabète » que le prétendait ce chanteur. Sous la dynastie des Qing et au début du xx e siècle, les livrets manuscrits de chants circulaient parmi les chanteurs. Ils dénotent une influence littéraire directe sur le répertoire et démontrent qu'au moins certaines personnes savaient lire et écrire dans la communauté des chanteurs. De nombreux livrets furent brûlés sous l'occupation japonaise du Jiangsu pendant la Seconde Guerre Mondiale, d'autres furent confisqués par les cadres du parti communiste au début des années 1950 ou détruits pendant la Révolution Culturelle. De cette façon, une grande quantité de ces textes disparut, parmi lesquels des épopées uniques de plusieurs centaines de vers. A différents moments de l'histoire, le fait de posséder des recueils de chansons, surtout si les poèmes décrivaient des scènes érotiques ou amoureuses, pouvait mettre la vie de leurs propriétaires en danger. L'ère du communisme ne fut certainement pas la seule période où les fonctionnaires gouvernementaux censurèrent les textes folkloriques dans la région de Wu. 

chinoise. Elle a sans aucun doute affecté le développement de la tradition du chant populaire durant les siècles passés. Le déclin actuel des chants populaires Wu résulte dans une certaine mesure de l'apogée du processus de la censure et des changements imposés à la culture dès l'époque impériale. Il ne découle pas uniquement de l'action de tel courant politique au vingtième siècle.

Les changements complexes survenus dans l'organisation agricole chinoise sont un autre facteur qui a décidé du sort du chant populaire dans le sud du Jiangsu. Jusque dans les années 1930, des groupes spéciaux de chanteurs, appelés shan'geban dans certaines régions, accompagnaient souvent les travaux des champs. Les propriétaires terriens les payaient d'habitude en argent liquide ou en nourriture et ils prenaient part à la récolte ou au repiquage du riz. Ils chantaient aussi pendant les foires organisées par les temples et animaient, en privé, les réceptions chez les riches. Avec le temps, certains shan'geban acquirent un statut de semi-professionnels et leurs principaux chanteurs élaborèrent des techniques vocales étonnantes. Après la redistribution des terres par les communistes en 1951, les shan'geban se dispersèrent petit à petit. Ils ne retrouvèrent plus leur gloire ancienne après les campagnes de collectivisation agricole qui eurent lieu dès 1958. On peut se faire une vague idée de ce que fut leur musique en écoutant par exemple les complexes chants de groupes que l'on trouve parfois encore dans la région de Jiashan.

Après avoir ainsi donné un bref aperçu de la culture du chant populaire $\mathrm{Wu}$, je présenterai les aspects pratiques de mon travail: la recherche des chanteurs, l'organisation des séances, le contact avec les participants, les problèmes rencontrés ainsi que les buts et les méthodes de mon enquête.

\section{A la recherche des chanteurs}

Tout d'abord, j'ai pris contact, à Suzhou, avec la Société pour la recherche sur le chant populaire $\mathrm{Wu}$ : celle-ci a en effet acquis une grande expérience dans la collecte des chants locaux et compte d'excellents chanteurs parmi ses membres. La société me fournit les noms et les adresses de nombreux chanteurs, et me donna de précieux enregistrements d'une sélection de chants enregistrés localement. Cette manière de procéder se révéla plus efficace que d'errer simplement dans la campagne, comme je l'avais fait en novembre 1988, lorsque j'avais parcouru (à bicyclette) avec mon compagnon Frank Kouwenhoven la région de Dongdongtishan pendant une semaine. Nous avions eu beau visiter tous les villages de la péninsule de Dongshan et de l'île de Xishan situées dans le lac Tai et nous renseigner dans chaque maison de thé et à chaque carrefour, aucun chanteur ne s'était jamais présenté. Nous leur avions pourtant chanté un grand choix de notre propre répertoire de chansons folkloriques et nous avions largement offert à boire à notre auditoire! Ce genre de convivialité donne d'excellents résultats dans les cafés et sur les places de villages balkaniques, mais elle ne fonctionne absolument pas dans la province du Jiangsu. Les paysans s'amusaient, mais ils ne chantaient pas en retour. Néanmoins, les visites à Dongshan et à Xishan ont été utiles pour d'autres raisons. Elles nous ont permis d'observer le déroulement de la récolte (auquel se réfèrent souvent les textes des chansons) et d'enregistrer sur le terrain les cris poussés par les transporteurs de riz. Ces cris ne sont pas des chants dans le sens de formes mélodiques développées, ceux qui les émettent ne les considèrent d'ailleurs pas comme telles, mais ils sont intéressants en tant que rudiments du folklore musical. 
ement, l'endroit approprié pour s'informer sur les chanteurs populaires est le bureau de la culture local. Le gouvernement chinois en a installé à tous les échelons, dans les grandes villes, dans les plus petites villes et dans les unités villageoises. Créés dès 1950 par le ministère de la culture qui les contrôle, ces bureaux maintiennent des contacts à tous les niveaux avec les branches correspondantes des associations chinoises de musiciens. La collecte des chansons populaires est une de leurs tâches mais elle n'est certainement pas leur unique ni même leur principale occupation. Ces bureaux organisent la propagande politique, préparent les affiches sur la planification des naissances ou sur les peines infligées aux criminels; ils se chargent en outre des divertissements : bals, théâtre ou jeux d'enfants. De même ils dirigent les bibliothèques et exécutent des travaux administratifs pour aider le gouvernement de la province. La collecte, l'édition et la réécriture des chants populaires était une de leurs tâches principales pendant la période de la propagande communiste intensive sous Mao Zedong. Ce travail de collecte est redevenu important ces dernières années depuis qu'on s'est rendu compte qu'une grande partie de la culture du chant populaire de la région de Wu (ainsi que d'autres régions du Jiangsu) était menacée de disparaître dans les deux ou trois prochaines décennies. Pourtant, la collecte des chants populaires ne semble pas être, à l'heure actuelle, une des tâches spécifiques des bureaux de la culture de la région de Wu; beaucoup d'entre eux ont d'autres priorités : par exemple, imprimer des étiquettes pour des pots de confitures et pour d'autres biens de consommation.

21 Au niveau du village, ce sont en principe les bureaux de la culture (wenhuazhan) qui fournissent aux visiteurs étrangers des informations sur les chanteurs locaux. Il peut s'avérer utile de connaître à l'avance les noms des chanteurs. Une lettre d'introduction (d'une institution d'études supérieures, conservatoire ou université) peut arranger les choses, mais j'ai rarement eu besoin de m'en servir. Les «cadres » de ces bureaux, en général des paysans très hospitaliers, sont fiers de l'intérêt que l'on porte à leur folklore.

J'ai l'impression qu'à un niveau plus élevé, comme dans les villes et les régions urbaines, les bureaux de la culture (appelés ici wenhuaju) sont entravés par la bureaucratie et la désorganisation et, en règle générale, sont un obstacle plutôt qu'une aide. Je suis allée deux fois dans le wenhuaju de Changshu pour demander l'autorisation de faire mon travail de recherche sur les chants populaires dans les environs de cette grande ville et j'étais munie de plusieurs lettres d'introduction qui ne m'ont pas permis pour autant de rencontrer les chanteurs: ces lettres étaient qualifiées d'«insuffisantes» malgré la reconnaissance officielle de mon projet par la Commission chinoise de l'éducation nationale. Les chercheurs chinois rencontrent eux aussi le même problème lorsqu'ils veulent collecter les chants populaires dans les campagnes : ils se heurtent souvent à un refus. Le meilleur moyen pour éviter ce genre de problème, c'est de prendre directement contact avec les stations de la culture du village et de visiter les villages, si possible sans préavis officiel, comme je l'ai fait plusieurs fois. Il n'est pas illégal d'enregistrer les chants populaires, de voyager dans la région de Wu et de rencontrer ses habitants. En définitive, la réussite d'une telle entreprise dépend surtout des bonnes relations, guanxi, que l'on entretient avec la population villageoise. Le mot guanxi ne doit pas être pris dans un sens péjoratif. Moi-même j'ai toujours essayé de détendre l'atmosphère et de mettre à l'aise les participants : la sensibilité aux conditions locales vaut souvent mieux qu'une lettre de recommandation formelle. 


\section{L'organisation des réunions avec les chanteurs}

23 proposait toute l'aide nécessaire pour trouver un logement pendant mon séjour (d'habitude un simple dortoir pour voyageurs, appelé zhaodaisuo) ou pour disposer d'une escorte officielle de police quand on le jugeait nécessaire. La police locale était gentille et serviable. Quelquefois, mais pas toujours, je devais annoncer ma présence dans le village, mais ce n'était qu'une simple formalité. Une fois, la police me servit même spontanément d'interprète. Personnellement, je n'ai jamais eu de mauvaise expérience avec les autorités de la police pendant mon enquête dans le Jiangsu, mais je sais qu'en Chine cela peut varier d'une région et d'un sujet de recherche à l'autre. Par exemple, dans la province de Hebei, des musicologues chinois et étrangers, qui étudiaient sur le terrain la musique locale taoïste et bouddhiste, se sont souvent heurtés à la résistance et au refus net de la police villageoise lorsqu'ils voulaient enregistrer les services religieux. Là encore les lettres d'introduction officielles n'étaient d'aucune utilité. Malheureusement, il semble qu'il y ait de grands problèmes de communication entre le ministère de la culture et le ministère de la sécurité publique.

Le secret qui entoure traditionnellement certaines musiques villageoises crée parfois un problème supplémentaire lorsqu'on veut recueillir la musique instrumentale. Ainsi, peu de chercheurs chinois ou étrangers ont pu enregistrer, en plus de trente ans, le zhoushan luogu, un genre musical de percussion de l'île de Zhoushan, située au nord de la province de Zhejiang (à l'est de l'aire dialectale Wu). Ceux qui ont entendu cette musique la trouvent fascinante; mais les musiciens refusent de se laisser enregistrer ou demandent un prix exorbitant pour les enregistrements, sachant que personne ne pourra les payer. On dirait qu'entraînés par leur orgueil professionnel, ils préfèrent cacher au monde extérieur la technique de leur art unique. Les raisons de ce mystère ne sont pas claires mais, partout dans le monde, il existe des musiques «secrètes", cette réserve étant probablement aussi liée à certaines fonctions magiques de la musique.

Les chanteurs populaires de la région de Wu ne cachent rien de leur musique et, sauf de rares exceptions, ne demandent pas à être payés. Au début du siècle, les meilleurs chanteurs des shan'geban recevaient occasionnellement de l'argent pour leurs prestations. Aujourd'hui, les chanteurs de rues et ceux qui offrent leurs services à l'occasion de mariages et de funérailles demandent un salaire. En outre certains collecteurs chinois de chants populaires se sont mis, depuis quelques années, à rémunérer les chanteurs pour leur travail, la représentation n'étant parfois qu'un simple prétexte pour aider les chanteurs. Il existe en effet de vieux paysans extrêmement pauvres qui ont besoin de soutien financier. En général je ne payais pas les chanteurs pour mes séances d'enregistrement, mais lorsque la situation l'exigeait je faisais des exceptions. Je découvris rapidement qu'une excellente façon de payer était de photographier les chanteurs et leurs voisins et de leur envoyer leurs portraits. Ceci est probablement le moyen le plus convenable et le plus acceptable pour remercier les villageois de leur coopération. De plus j'offrais aux musiciens et à leur auditoire la possibilité d'entendre les chansons qui venaient d'être enregistrées. Et souvent je chantais à mon tour. Les chanteurs et les autres villageois attachaient une grande importance à cela et leurs commentaires sur la musique se révélaient très intéressants. 
26 La plupart des séances de chants étaient organisées par les wenhuazhan; parfois elles avaient lieu dans le bureau de la culture et non chez les chanteurs. Je devais souvent insister sur le fait que les conditions de pauvreté des familles ne me rebutaient pas et que je préférais enregistrer les chanteurs chez eux. D'habitude les fonctionnaires acceptaient cela, mais une fois un chanteur m'a littéralement suppliée de ne pas aller chez lui, disant qu'il n'avait même pas une chaise pour me recevoir.

En général, un petit nombre de chanteurs (trois au maximum) participait à la séance, les meilleurs sessions étant celles où je pouvais me concentrer sur une seule personne.

Mais avant d'expliquer comment se déroulaient effectivement les séances d'enregistrement, j'aimerais mentionner certaines limitations à ma liberté de mouvement sur le terrain.

\section{Transport, communication et conditions climatiques}

Je savais d'avance qu'il ne suffirait pas d'écouter et d'enregistrer un chanteur populaire une seule fois. J'espérais pouvoir recueillir plusieurs fois les mêmes histoires et chansons et poser autant de fois que possible les mêmes questions pour déceler ce qui variait et ce qui restait cohérent dans les prestations musicales et dans les discours des chanteurs. Mais il m'était difficile de me tenir à ce principe à cause des nombreuses limitations et restrictions pratiques que je rencontrais.

Dans la plupart des villages, je ne pouvais pas séjourner pendant de longues périodes (des semaines ou des mois). D'habitude, je ne pouvais rien entreprendre sans la supervision des cadres locaux, mais je n'étais pas en mesure de les payer pour leur soutien et leur coopération. Il ne me restait qu'une solution : rendre des visites brèves et répétées à un certain nombre de villages, en réduisant la durée normale de mes séjours à un ou deux jours seulement. Cette solution me semblait acceptable parce que je ne me proposais pas d'étudier, comme certains anthropologues, la dynamique des processus sociaux en cours, ce qui aurait exigé une présence à long terme. Les chansons n'étaient interprétées que parce que (et lorsque) je venais le demander.

31 Cependant, même ces visites courtes et rapides me posaient des problèmes. En Chine, les services de la poste sont lents et peu fiables; certains de mes contacts officiels, comme les wenhuazhan, n'avaient pas de téléphone. Ainsi, beaucoup de messages et de questions que j'avais envoyés de Shanghai n'étaient jamais arrivés à destination. Je pouvais donc difficilement déterminer à l'avance si le moment que j'avais choisi pour ma prochaine visite conviendrait. A plusieurs reprises j'ai voyagé pour rien, parcourant plus de cent cinquante kilomètres pour trouver les chanteurs les plus importants malades, absents ou occupés à des besognes qu'ils ne pouvaient interrompre. Quelquefois, le télégramme fit des miracles. Mais dans l'ensemble, je ne pouvais rien contre ces problèmes de communication, il fallait seulement que j'en tienne compte. Ceci valait aussi pour le trafic et le transport public. En bus, bateau, camion, taxi, cyclo-pousse ou à vélo, ma vitesse de croisière était en moyenne de $15 \mathrm{~km} / \mathrm{h}$ dans la région de Wu. Lorsque je devais emprunter plusieurs moyens de locomotion pour atteindre un endroit, je passais la journée entière sur les routes. Il était possible de rouler à bicyclette, mais l'expérience était éprouvante à cause des mauvaises routes, de la pollution de l'air et de la poussière.

32 J'aurais pu éviter d'autres problèmes pendant mes recherches. Le milieu de l'hiver et la période chaude de l'été ne conviennent pas à l'enquête prolongée sur le terrain dans le 
Jiangsu. J'ai commis une grave erreur en travaillant principalement en hiver dans cette région. D'octobre 1988 à février 1989, la région de Shanghai fut frappée par la plus grande sécheresse du siècle, à laquelle s'était jointe une longue période de gel, en décembre et en janvier. Les maisons ne sont pas chauffées dans la campagne chinoise, au sud de la rivière Yangzi. Bien que je me fusse habillée en conséquence, j'avais sous-estimé l'effet désastreux des gels prolongés sur ma condition physique et mon énergie. Pendant cette période, mon enquête se déroulait au rythme caractéristique d'« une semaine à la campagne, une semaine au lit ». La rigueur de l'hiver influençait aussi l'état général des chanteurs et affectait leur voix.

Il y avait un autre problème lié aux rigueurs du climat: pour les cadres de certains wenhuazhan, les pluies diluviennes marquaient le signal d'arrêt général de toutes les activités extérieures. J'eus de la peine à trouver un bateau pour aller dans un des villages pendant la saison des pluies; mais finalement mes efforts furent bien récompensés. Les chanteurs de la région de $\mathrm{Wu}$ sont toujours prêts à chanter et les vieux ont beaucoup de temps, sauf à l'époque de la récolte ou des semailles (nongmang, fin octobre-début novembre et en mai-juin), où ils sont censés aider leur famille dans les travaux des champs ${ }^{7}$.

Bref, une bonne planification était d'une importance capitale, et pas seulement en termes d'événements saisonniers. Il ne servait à rien d'arriver à 11 heures du matin dans un village puisque c'était le début de la sieste. Pour rencontrer les chanteuses, je devais tenir compte du fait qu'elles devaient préparer le repas et quitter la séance encore plus tôt dans la matinée. D’habitude, je décidais sur place si j'allais ou non passer la nuit dans un village pour continuer la séance le lendemain.

Fig. 3 : L'auteur discute de chants populaires avec le chanteur Jin Wenyin dans le village de Shengpu.

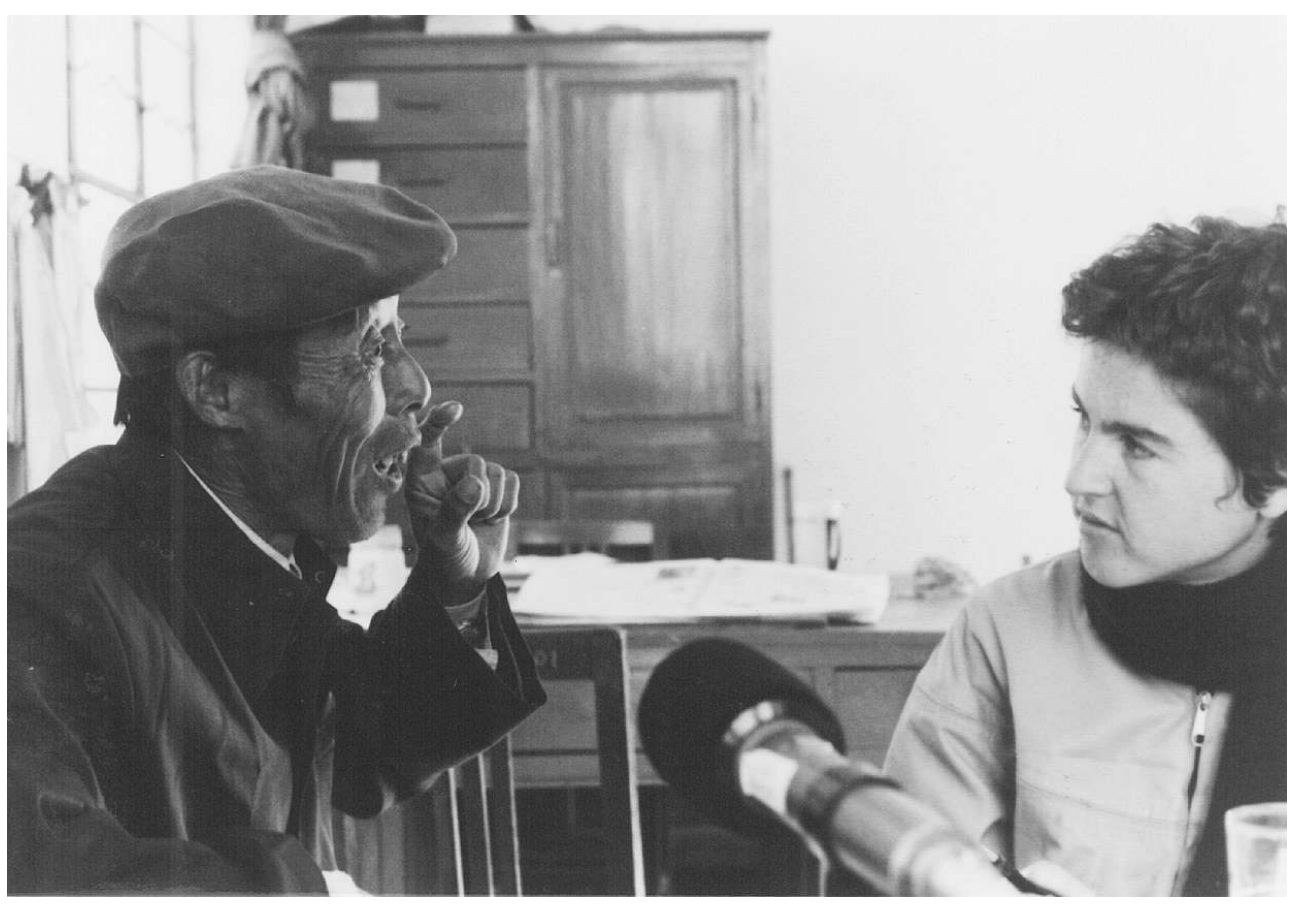

Photo : Frank Kouwenhoven. 


\section{Les séances d'enregistrement} entretiens et des séances d'enregistrement. Je m'efforçais de ne pas fatiguer les participants, mais je sous-estimais à quel point mes visites les excitaient parfois. Deux chanteurs souffrant de surmenage tombèrent malades après mon départ. En principe, c'était aussi au personnel des bureaux de la culture de tenir compte de la condition physique des chanteurs, mais je pense que souvent on ne se rendait pas compte du rôle central que les chanteurs jouaient dans mes séances d'enregistrement. Voilà en quoi consistait la difficulté principale de ces entretiens. De nombreux fonctionnaires ne traduisaient pas les questions que je posais aux chanteurs mais répondaient plutôt à leur place. Il pensaient probablement que leur longue expérience dans le domaine de la culture du chant local justifiait cela ; ils croyaient peut-être aussi qu'ils étaient capables de formuler leurs points de vue mieux que des paysans illettrés. Cette attitude provoquait souvent des discussions entre les chanteurs et les fonctionnaires.

Parfois un spécialiste des chants populaires du conservatoire de musique de Shanghai m'accompagnait dans mes expéditions et me servait d'interprète pour les différents dialectes de la région de Wu. Mais en général, je devais compter avec les services du personnel des bureaux de la culture, ce qui n'était pas toujours idéal.

Il me fut difficile d'apprendre en peu de temps à parler couramment ces dialectes villageois, si différents les uns des autres. Mais à force d'écouter, avec l'aide d'interprètes locaux, les enregistrements et les entretiens, je finis par comprendre plusieurs aspects de ces dialectes. A chaque étape de mon travail, j'essayais de communiquer directement avec les chanteurs. C'était difficile de leur expliquer mes intentions et d'obtenir des informations fiables. Les problèmes de dialectes et de mauvaises interprétations n'étaient pas la seule cause de difficulté, il y avait aussi de leur part un problème de politesse. Les villageois ne voulaient pas mentir, mais, croyant que j'attendais d'eux certaines réponses, ils se faisaient une joie de me satisfaire, souvent en se contredisant. Voici un extrait, parmi beaucoup d'autres, d'une conversation qui illustre le genre de problème que cela soulevait. J'avais demandé à un chanteur, Jin Wenyin, ce qu'il pensait du shan'ge; sa réponse me laissa quelque peu perplexe :

Jin : - « On doit toujours distinguer les chansons populaires des chansons d'amour. Les chansons d'amour sont plus longues que les chansons populaires [...]. Quand j'étais jeune, les jeunes filles les chantaient doucement, le soir, à la maison, derrière les portes closes. Elles ne chantaient qu'en cachette» (Il murmure un vers pour mimer la timidité de la jeune fille). «Aujourd'hui, les filles ont un autre comportement. Parfois, elles portent même une robe! Il y a deux jours, une fille du village s'est mariée. Elle ne portait pas de pantalons, mais une robe. Voyez-vous, cela fait jaser! Les jeunes mariées devraient porter des pantalons et des vestes molletonnées, qu'il fasse froid ou chaud.

- Mais comment décrivez-vous le shan'ge?

- Ce sont des chansons populaires, chantées en dialecte local. Chaque région a son propre shan'ge. D'habitude, elles ont 4 vers, rarement 8 à 12 vers. Oui, on appelle aussi shan'ge les plus longues, mais si on devait les classer par genres, les longues chansons sont en fait des chansons d'amour ». (il chante une chanson à propos des produits alimentaires locaux, puis s'interrompt) : «Ce genre de chansons ne vous intéresse pas, parce qu'elles ne parlent pas d'amour et elles ne sont pas des chansons populaires. 
- Pourquoi dites-vous que ce ne sont pas des chansons populaires?

- Eh bien,oui! ce sont aussi des chansons populaires, mais pas des chansons

d'amour.

- Est-ce qu'on les appelle aussi shan'ge?

- Oui, on les appelle aussi shan'ge. Mais parfois on les classe dans la catégorie des

chants d'opéra local. »

38 pour distinguer ce qui est valable de ce qui n'a pas de sens. Quand c'était possible, je reprenais l'entretien avec un questionnaire à portée de main. Mais j'utilisais la liste des questions avec discrétion pour ne pas donner l'impression d'un «interrogatoire». Certains chanteurs se seraient trop souvenu des contre-interrogatoires et des séances de critique qu'ils avaient subis pendant la Révolution Culturelle.

La plupart des séances se déroulaient à l'intérieur des maisons, les chanteurs et les interprètes étant assis autour d'une table. Pour des raisons pratiques, je travaillais avec deux enregistreurs, placés tous deux devant le chanteur. Un des enregistreurs avait un microphone relativement imposant, il enregistrait les chansons "officielles »; l'autre, plus petit, tournait sans arrêt, enregistrant les discussions et toutes les chansons qui étaient interprétées sans être annoncées auparavant; par exemple, lorsqu'un chanteur commentait de façon spontanée quelque chose qui s'était passé dans la chambre, ou s'il (ou elle) avait envie de chanter un poème érotique qui n'aurait pas été considéré comme une chanson "officielle " pendant la séance d'enregistrement. Les bandes enregistrées des entretiens m'ont aidé à conserver beaucoup de ces précieux moments. De retour chez moi, j'écoutais plusieurs fois de suite les discussions, en présence d'un deuxième interprète qui contrôlait les traductions des interprètes précédents et ajoutait ce qui manquait.

Pendant les deux premières années de mon travail de terrain, je ne demandai pas aux chanteurs d'exécuter des chansons précises. J'espérais découvrir plus au sujet de la position et de la signification particulières du shan'ge, en laissant les chanteurs interpréter ce qu'ils préféraient. Plus tard, j'eus des exigences plus spécifiques, notamment par rapport aux textes. Je demandais des répétitions pour rassembler des matériaux me permettant de faire des comparaisons.

41 Mon compagnon, Frank Kouwenhoven, surveillait l'équipement d'enregistrement du son, la vidéo et l'appareil photo, me permettant ainsi de me concentrer sur les entretiens. Cette façon de partager le travail se révéla très pratique. Sans une telle assistance, les séances auraient été chaotiques.

Ne pouvant compter sur les systèmes d'alimentation en électricité dans les villages où il y a de fréquentes coupures de courant et des variations de voltage, nous devions transporter des kilos de piles et recharger souvent nos appareils, même pendant les courts voyages. Le contrôle du volume sonore était difficile parce que certains chanteurs commençaient à chanter très fort mais continuaient plus doucement pour ménager leur voix (beaucoup d'entre eux se vantaient d'ailleurs d'avoir chanté bien plus fort dans leur jeunesse). Les enregistrements vidéo, que nous avons réalisés à plusieurs reprises, posaient des problèmes techniques particuliers d'éclairage, de son, de mise au point et de cadrage. Seule, je n'aurais jamais pu combiner des tâches aussi diverses. 

démonstrations. Elles montraient clairement que les représentations se passaient dans une atmosphère détendue. Elles prouvaient que les dialogues que je menais d'une façon expérimentale avec un certain nombre de chanteurs gardaient un caractère informel et décontracté et n'étaient pas considérées comme des «intrusions" artificielles, que personne n'avait sollicitées, dans la culture locale du chant. Elles révélaient et permettaient de sauvegarder certains aspects des séances de chant qui n'apparaissaient pas dans les enregistrements sonores comme l'ordre dans lequel les voix individuelles chantaient dans le shan'geban, des phénomènes particuliers comme le rapide tremblement du pied, auquel les chanteurs avaient automatiquement recours lorsqu'ils chantaient le shan'ge, ou le fait qu'il y avait des auditeurs qui remuaient leurs lèvres en accompagnant de manière synchronique les paroles des chanteurs, prouvant par là qu'ils connaissaient les chansons (même s'ils prétendaient le contraire).

J'ai consacré un certain temps à filmer différents types de travaux d'intérieur et de travaux des champs qui jouaient un rôle dans les textes des chansons. Cela m'a beaucoup aidée lorsque j'ai commencé à traduire les chansons. De même, j'ai pu montrer aux spécialistes des chants populaires à Shanghai des extraits où j'avais filmé les chanteurs, obtenant ainsi leurs commentaires sur les aspects contextuels des chants exécutés. A ma demande, des chanteurs me montraient les fonctions spécifiques des chansons, telles qu'elles avaient lieu dans le passé durant les travaux d'extérieur. Dans l'île de Chongming, les pêcheurs faisaient entendre leurs haozi (cris de travail) en les accompagnant de mouvements de prise ; à Zhaozhaiqian, les porteurs de pierres chantaient en poussant leurs charrettes; dehors, les paysans représentaient les shan'ge et les chants de travail en portant des charges et en travaillant dans les champs. C'était la vidéo qui saisissait le mieux ces mouvements corporels associés à l'expression musicale. J'ai photographié à peu près tous les chanteurs et les objets spécifiques, outils, vêtements, nourriture et meubles dont ils parlaient dans leurs chansons. Les photos et les bandes vidéo constituent un document sur les chanteurs et leurs traditions dont la valeur dépasse la simple recherche. Elles sont utiles parce qu'elles me rappellent l'atmosphère générale dans laquelle se déroula ma recherche sur le terrain et elles ont capté des événements que je n'avais pas notés dans mes cahiers.

Très peu de chanteurs étaient gênés par la présence du micro ou de la caméra. La plupart d'entre eux gardaient un visage immobile. Dans l'ensemble, ils trouvaient plus facile de chanter que de répondre aux questions - avec ou sans caméra en marche. Certains étaient très enthousiastes à l'idée que leurs représentations seraient "télévisées en Europe ", c'est du moins ainsi que quelques fonctionnaires locaux interprétaient l'utilisation de la vidéo. Je tâchais d'expliquer en termes simples le but de mes recherches aux chanteurs. Les questions d'analyse textuelle et musicale de leur répertoire devaient probablement leur échapper, mais dans l'ensemble leurs réactions face au projet étaient positives, surtout lorsqu'ils réalisaient que c'était une façon de sauvegarder leur répertoire. Ces mots d'un chanteur, Zhao Yongming, résument bien ce que beaucoup d'autres disaient :

«Mè hao! (très bien!) De cette façon, les générations futures sauront que ces précieux chants ont existé. Regardez-moi ! J'ai 70 ans. Dans quelques années je serai mort. Si mes chansons ne sont pas enregistrées, personne ne s'en souviendra dans un avenir proche.»

J'avais de la peine à convaincre les cadres des wenhuazhan que je ne m'intéressais pas seulement aux « meilleurs » chanteurs de leurs villages, mais à tous ceux qui étaient prêts 
à chanter. Je ne réussissais pas toujours à les persuader de me présenter les «moins bons » chanteurs, mais les séances d'enregistrement attiraient une grande audience et révélaient parfois, parmi les auditeurs, d'autres chanteurs qui avaient d'habitude un répertoire plus limité. En général, leurs commentaires et les remarques des spectateurs s'avéraient importants (les images vidéo me permettant parfois de découvrir qui parlait). D'un point de vue pratique ou technique, les entretiens se déroulaient le mieux sans audience, en présence de quelques proches parents seulement. Mais en général cela n'était pas possible. On avait beau fermer les portes (lorsqu'il y en avait) cela n'empêchait pas les gens de les ouvrir. Dans les villages, les maisons privées sont en fait des «places publiques ", du moins pendant la journée, et surtout lors d'un événement inattendu comme la visite d'un étranger.

La présence d'un grand nombre de spectateurs avait le désavantage évident de produire beaucoup de bruits de fond, surtout des cris d'enfants. Dehors, il y avait aussi beaucoup de bruit. Près des voies publiques, la campagne est loin d'être silencieuse; dans la région de Wu, le trafic intense a gâché un certain nombre d'enregistrements.

\section{Les thèmes-clés et les méthodes de recherche} les aspects de la culture du chant populaire $\mathrm{Wu}$, qui s'étendaient encore devant moi comme une terra incognita. Il existait peu d'études régionales complètes consacrées au chant populaire chinois et je pensais que ma tentative fournirait un cadre général pour les recherches à venir. Cela me semblait plus urgent que de me concentrer sur des aspects limités de certaines chansons, même s'il était clair dès le début que la musique et la langue du shan'ge se trouveraient au centre de mon étude.

$\mathrm{Au}$ début de mon enquête, les méthodes globales de recherche que Brăiloiu avait appliquées en Roumanie pour étudier les chanteurs populaires me servirent de modèle (1984a : 1-58 et 59-85). J'utilisais un questionnaire détaillé, j'avais préparé des dossiers individuels pour chaque chanteur, essayé de rassembler le plus d'informations possibles sur chaque interprète, traduit et transcrit in extenso les bandes d'enregistrement des entretiens et recueilli les paroles de presque toutes les chansons enregistrées. J'avais envisagé la possibilité d'entreprendre l'étude détaillée d'un ou deux villages en suivant ces lignes. Mais je compris bien vite que cette approche n'était pas réalisable. D'une part, il restait trop peu de chanteurs dans la région de Wu pour pouvoir me concentrer sur un ou deux villages - à moins de passer des mois à la recherche de plus d'informateurs ${ }^{8}$; d'autre part, la région entière était trop grande pour pouvoir appliquer les méthodes de Brăiloiu. En interviewant tous les chanteurs, l'un après l'autre et à plusieurs reprises, et en déterminant l'étendue précise de leur répertoire, j'aurais prolongé le temps consacré à mon enquête au-delà de toute limite raisonnable.

Au lieu de cela, je décidai de me concentrer sur un petit groupe de chanteurs habitant dans différentes parties de la région. J'espérais construire petit à petit une image de la culture du chant populaire $\mathrm{Wu}$ à partir de leurs propos et de leurs exécutions. Les chanteurs n'étaient pas délibérément "sélectionnés " à cette fin. Ils se présentaient plutôt spontanément parce qu'ils étaient accessibles et relativement loquaces, que leur répertoire de textes de chansons était riche et ce qui était vraiment important, parce que je pouvais compter sur la présence d'interprètes fiables. Autour de ces informateurs-clés 
se trouvaient toujours d'autres chanteurs en qui je pouvais avoir confiance et qui m'aidaient à enrichir mes impressions, même s'ils étaient moins prolifiques que les artistes du chant et les mémoires vivantes de la poésie populaire. M'étant seulement rendue dans un petit nombre d'endroits de la région de Wu et n'ayant interviewé qu'un nombre limité de chanteurs, je courais le risque d'extrapoler une situation régionale à partir de données collectées au hasard dans des localités isolées. Mais, tout en révélant une très grande variété dans la culture locale du chant, les séances laissaient entrevoir une unité sous-jacente qui dépassait mes prévisions.

51 En constatant qu'il y avait une certaine unité dans le répertoire et les formes musicales, je fus amenée à étudier plus en détail ces aspects de la culture du chant populaire Wu et à restreindre le champ de mes recherches. Après avoir analysé les premières transcriptions musicales, je remarquai qu'il existait une certaine homogénéité mélodique entre les chanteurs d'un seul et même village. Les transcriptions ultérieures montrèrent que ceci était une caractéristique générale de la région de $\mathrm{Wu}$. L'existence de plusieurs « régions mélodiques " m'incita à faire une recherche comparative. Je puisai dans les études analytiques de Bayard sur les mélodies populaires anglo-américaines (1939:122 et suiv.) l'inspiration et les idées nécessaires à une approche musicologique appropriée. Le contraste entre le caractère "supra-régionale » des textes et l'étendue géographique beaucoup plus limitée des mélodies posait un problème majeur. Il fallait donc étudier de plus près les dichotomies et les concordances entre la langue et la musique. Je me concentrai spécialement sur les formules mélodiques qui existaient dans la musique et qui correspondaient aux formules textuelles dans les poèmes (bien que placées à des endroits différents). Je trouvai dans le livre classique d'Albert B. Lord, The Singer of Tales (1976), et dans l'important article de Leo Treitler, « Homer and Gregory, the transmission of epic poetry and plainchant » (1974:333-372), les outils qui me permirent d'étudier ces formules musicales et textuelles, tout en reconnaissant les limites manifestes de la théorie orale de Lord et de Parry ${ }^{9}$. Je n'eus pas assez de temps pour trier les formules des textes des chansons populaires Wu à une grande échelle. Mais je décidai de faire une analyse comparative d'un nombre réduit de variantes textuelles. En me plongeant dans la théorie orale je relus avec bonheur l'Odyssée d'Homère dans une perspective « formuliste ». Cela me permit d'équilibrer mes vues sur la structure des longues chansons épiques $\mathrm{Wu}$.

L'étude des phrases toutes faites, dans la musique comme dans les textes, soulevait naturellement des questions relatives à l'oralité et à l'écriture, ainsi qu'au processus de création dans les chansons. Je compris qu'il existait, dans les traditions du chant Wu, une interaction féconde entre la culture populaire et la culture de l'élite, et entre les matériaux oraux et écrits.

Les remarques de Ruth Finnegan sur l'individualité et la créativité dans la littérature orale furent très stimulantes lorsque j'ai commencé à étudier de plus près la poésie des chansons (1988 :surtout 63-77). Les poèmes contenaient une telle variété de thèmes que je ne pouvais espérer les examiner tous. Je m'intéressais entre autres aux chansons qui décrivaient les chanteurs et leur art du chant. A un certain moment, cela aboutit à la recherche de personnages qui étaient nommés dans les poèmes et dont les noms se référaient à des gens ayant jadis vécu dans la région de Wu. L'un d'entre eux était Zhao Shengguan, un chanteur semi-légendaire dont les descendants vivent à Zhaozhaiqian, un village situé à l'ouest de Suzhou. Ma tentative de relier le mythe à la réalité, en me rendant dans ce village, se révéla instructive à maints égards. 

d'introduire brièvement ici quelques localités-clés de ma recherche. La première région où j'ai recueilli des chansons fut Wuxian, le district qui entoure Suzhou. Pendant plusieurs années, j'ai visité douze fois les villages de Shengpu et Huangdai, me concentrant surtout sur deux chanteurs, Lian Dagen et Jin Wenyin. Au cours de mon enquête, je rassemblai des matériaux de quinze autres chanteurs de ces villages. Dans d'autres parties de Wuxian, je me rendis à Zhaozhaiqian, puis dans la péninsule de Dongshan et sur l'île de Xishan (lac Tai). Au nord-est, j'ai étendu le rayon de mes investigations en séjournant brièvement dans deux villages du Kunshan.

Huangdai (16 km au nord de Suzhou) peut être considéré comme un modèle de communauté d'agriculteurs et de pêcheurs de la municipalité de Wu. Il s'agit en fait d'un conglomérat (xiang) de vingt trois petits villages (cun) ayant en tout 44000 habitants. Du centre de Huangdai - rue étroite et animée, bordée de vitrines de magasins - jusqu'aux villages périphériques, il faut compter une demi-heure à bicyclette, à travers les rizières. Plusieurs cun (comprenant chacun quelques centaines de cabanes et de maisons) bordent le lac Cao (Caohu). Jadis, on pouvait entendre les cultivateurs et les pêcheurs chanter le shan'ge le long des berges de ce lac peu profond. Les personnes âgées se rappellent encore comment l'eau portait loin les voix des chanteurs. Jusque dans les années 1960, les rives du lac Cao étaient couvertes d'arbres et de buissons qui ont été coupés pendant le Grand Bond en avant (1958-1959) afin de servir de combustible pour les hauts fourneaux. Les canards et les oies disparurent du paysage en même temps que les arbres et les versants escarpés du lac s'écroulèrent. Le lac Cao regorgeait de crabes et de crevettes; mais les changements écologiques forcés, liés ces dernières années à une exploitation excessive du 
lac par les entreprises d'Etat, épuisèrent ses ressources et obligèrent beaucoup de monde à quitter la pêche pour l'agriculture. Les longues rangées de maisons à deux étages attestent la vitalité économique de la région, due en grande partie au développement agricole et à l'artisanat ; mais le passé n'est pas oublié. Les villageois m'ont dit : « De nos jours les gens ne pensent qu'à manger, à beaucoup manger». La région a souffert des grandes famines et les taxes agricoles ont pesé lourdement sur les foyers locaux jusqu'au milieu des années 1980.

Il y a encore des pêcheurs dans le Huangdai et on les trouve en grand nombre dans quelques autres villages. Mais je n'ai jamais entendu de chants de pêcheurs (yuge) pendant toute mon enquête ; pourtant, j'ai trouvé plusieurs chansons se rapportant à la pêche qui avaient dû jadis faire partie du répertoire des pêcheurs. Je n'avais pas le temps d'étudier en détail ce genre musical mais j'ai reçu des informations sur les chants des pêcheurs du lac Tai par un fonctionnaire de la culture et collecteur de chansons, Ren Mei. Natif de la région située au nord du lac Tai qu'il visite fréquemment, il est lui-même un excellent chanteur. Je fis plusieurs petits voyages à Dongshan et dans l'île de Xishan, mais je n'ai pu enregistrer que des cris de travail dans les plantations d'orangers et près des chantiers de Luxiang, Jinwan, Jinqiao et Bingchangcun. Les grands bateaux de pêche avec leurs nombreux mâts et voiles, se tenaient loin sur le lac Tai, et je n'avais pas le temps d'enquêter dans les communautés de pêcheurs.

Après Wuxian, ma deuxième région-clé fut la municipalité de Wujiang, en particulier la région qui entoure Luxu (prononcé aussi Luqu en dialecte local). Luxu est une commune de vingt et un villages avec une population totale de 30000 habitants. Elle est située au bord du lac Fen (Fenhu). Son chef-lieu (7000 hab.) est le centre industriel et le marché de toute la région de Fenhu. Lieu de passage entre les provinces de Jiangsu (au nord-est), de Zhejiang (au sud) et du district de Shanghai (à l'ouest), il relie les villes industrielles de Jiashan, Wujiang et Qingpu. Selon les fonctionnaires de la culture, on parle quatre dialectes dans cette région, même si les shan'ge ne sont apparemment chantés que dans un seul et même dialecte (chose que je n'ai pas pu vérifier pendant mon enquête). Malgré le développement économique rapide, le taux d'analphabétisme est plus élevé dans la municipalité de Wujiang que dans les autres parties du Jiangsu ${ }^{11}$. C'est peut-être une coïncidence mais j'ai trouvé, dans cette région particulière, une grande concentration de chanteurs populaires qui subsistent encore. J'ai recueilli des chansons dans différents villages situés près de Luxu aussi bien que de Shenta, une plus petite communauté qui se trouve à six kilomètres au nord. Trois chanteurs, Zhao Yongming, Jiang Liansheng et Lu Fubao, m'ont servi d'informateurs-clés. J'ai ajouté à leurs chansons des matériaux provenant de douze autres chanteurs. J'ai poussé ma recherche vers l'extrémité nord du Zhejiang, juste de l'autre côté de la frontière provinciale : il s'est avéré que Dingzha et Taozhuang (onze chanteurs) partageaient les caractéristiques mélodiques du shan'ge solo que j'avais trouvés à Luxu.

Dans l'ensemble, Wuxian et Wujiangxian sont les municipalités que j'ai le mieux étudiées, en partie parce que j'ai pu compléter mes enregistrements de terrain par ceux des nombreux collecteurs de chants de ces régions.

61 D'autres régions plus petites où j'ai mené mon enquête comprennent deux communautés villageoises dans la municipalité de Changshu (Baimao et Shuangfeng), le village de Dongting dans la municipalité de Wuxi (important surtout à cause de Qian Afu, un de mes informateurs-clés) et certaines parties de l'île de Chongming (à l'embouchure du Yangzi). 
62 A Baimao, j'ai rencontré jusqu'à quatorze chanteurs différents dont la plupart étaient des jeunes filles, ce qui représentait une exception par rapport au schéma habituel. Il y avait eu apparemment dans cette région de grands festivals de chants organisés par le gouvernement. A Baimao, les jeunes qui aiment chanter sont «instruits » dans l'art du chant shan'ge par un maître d'école local qui compose de nouvelles chansons pendant ses heures de loisirs. Le plus grand changement survenu dans le shan'ge de la nouvelle génération, à Baimao, est la pétrification de sa forme musicale. L'air est chanté comme si on déchiffrait une partition, sans les habituelles variations mélodiques et les ornementations si caractéristiques du shan'ge interprété par les vieux chanteurs.

Après ces localités du sud du Jiangsu, j'ai visité un village situé au nord de cette province, Shuangdu, dans la municipalité de Rudong. En fait, j'y avais collecté des chants populaires en 1986 et je décidai d'y effectuer un bref séjour supplémentaire en 1990. Ce fut une expérience révélatrice. La langue, la musique et quelques autres aspects des chants populaires de Shuangdu différaient d'une manière étonnante de ce que j'avais trouvé dans la région de $\mathrm{Wu}$.

Fig. 4 : « Le roi des chanteurs », Qian Afu (82 ans) de Dongting, village près de Wuxi.

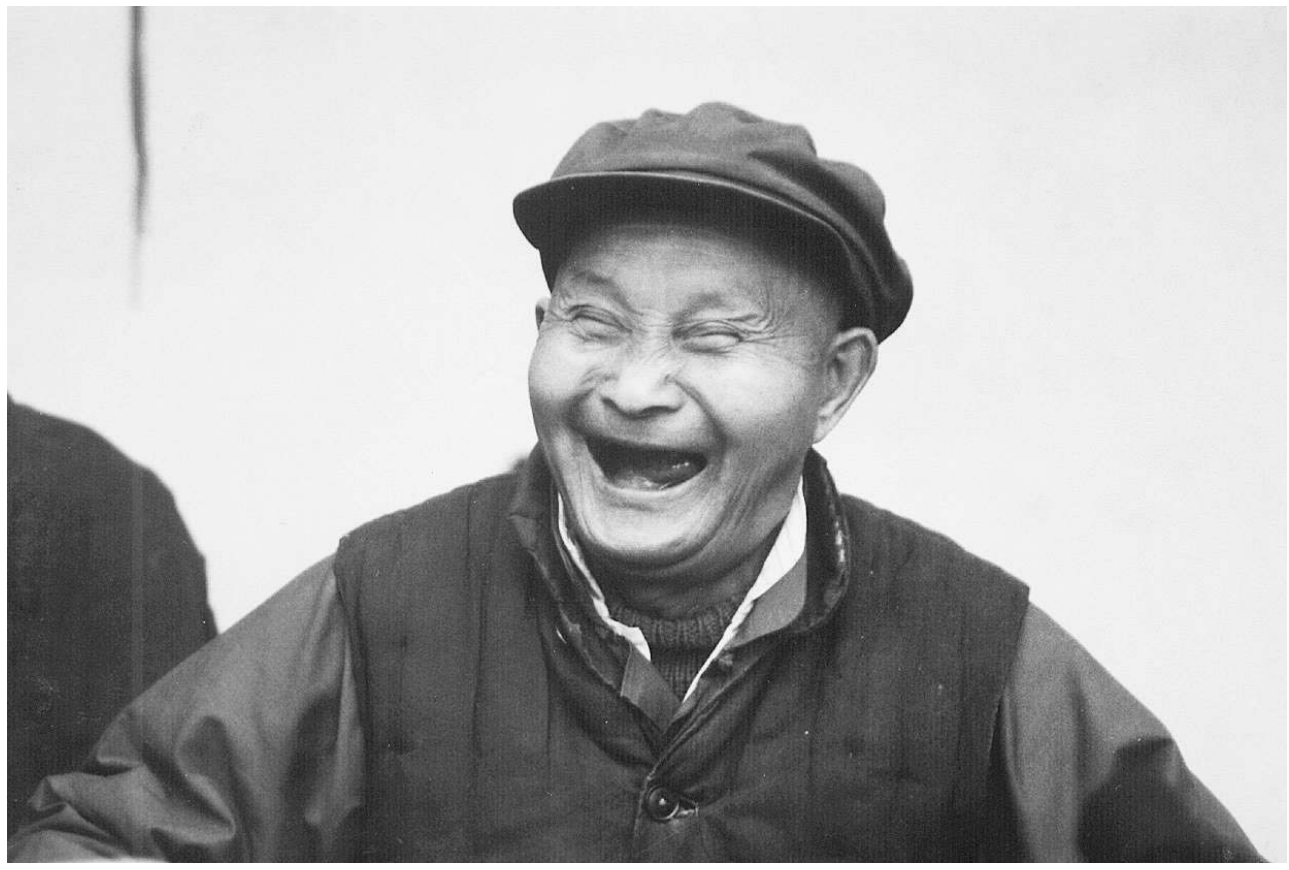

Photo : Frank Kouwenhoven.

\section{Matériaux et traitement de l'information}

En quatre ans, j'ai rassemblé environ deux cents bandes enregistrées de chansons et d'entretiens dont j'ai déposé des copies au Musée du folklore de Suzhou au bénéfice des chercheurs locaux. Les originaux sont actuellement conservés à Leiden, à la bibliothèque de Chime, pour la consultation générale. Ils comprennent les enregistrements d'environ sept cents chansons. J'ai étudié les chants enregistrés sur le terrain dans le cadre de deux cents autres versions de chansons et de fragments de chansons enregistrées par les chercheurs locaux auxquelles s'ajoutent des notations manuscrites de textes de chansons 
(compilées par les collecteurs locaux ou par les chanteurs eux-mêmes) et quelques anthologies de chants publiées localement et compilées dès les années 1950. Tous ces matériaux concernent essentiellement la région de $\mathrm{Wu}$.

Une grande partie des bandes enregistrées par les collecteurs locaux sont dans un état si défectueux qu'aucun traitement ultérieur du son ne pourrait les améliorer sans perdre les caractéristiques essentielles du chant. (Certains enregistrements ont été envoyés aux Archives Sonores Nationales de la Grande-Bretagne pour y être restaurés). Malgré cela, ces enregistrements locaux se sont avérés extrêmement précieux pour mes recherches.

J'ai transcrit, traduit et analysé pour mon étude une petite partie des chansons collectées. J'ai examiné les enregistrements des entretiens avec l'aide d'un (deuxième) interprète et j'ai trié les données significatives, pour lesquelles j'ai conçu les catégories suivantes: répertoire de mélodies (les airs), répertoire de textes (titres de chansons), terminologie des chansons, caractéristiques de la performance, contexte de la performance, festivals, rituels, variation et création, origine des chansons, contenu des poèmes, émotion, chants d'amour et chants érotiques, politique, esthétique, collecte des chants populaires, livrets (manuscrits) et tradition écrite, chants épiques, chants de groupe, shan'ge, haozi, xiaodiao, rythme, langue et dialecte, chants accompagnés, autres types de musique traditionnelle, localités. Les propos significatifs des chanteurs et des autres informateurs locaux qui avaient un rapport avec ces thèmes ont été classés en conséquence. Ensemble, ils forment la base de l'image globale de la culture du chant populaire $\mathrm{Wu}$, telle qu'elle est décrite ici.

En plus des chansons et des entretiens enregistrés, j'ai consulté plusieurs collections régionales de chansons. La Société pour la recherche sur le chant populaire Wu avait mis à ma disposition plusieurs anthologies de chansons $\mathrm{Wu}^{12}$ ainsi que des collections locales provenant des municipalités de Wuxi, de Wujiang et de Jiashan ${ }^{13}$, et avait attiré mon attention sur l'existence de l'excellente revue de littérature populaire Shanghai Minjian Wenyi Jikan (Revue trimestrielle d'art et de littérature populaires de Shanghai). En outre, j'ai recueilli quelques polycopiés produits localement et photographié plusieurs précieuses collections manuscrites que j'ai trouvées dans les villages. Quelques anthologies générales chinoises de chants populaires se sont révélées intéressantes en tant que matériaux de référence, en particulier les volumes «non-officiels » du Jiangsu qui font partie de la grande anthologie des chants populaires, recueillis par l'Association des musiciens chinois de Pékin. Une autre source utile furent deux volumes de chants populaires de la région de Suzhou, contenus dans une série générale sur la musique folklorique de Suzhou ${ }^{14}$.

Dans l'ensemble, il fut plus facile de trouver des études sur les textes et sur l'arrière-plan historique des chants populaires que sur leur musique. Aucune recherche ethnomusicologique valable n'ayant été entreprise dans le domaine des chants $\mathrm{Wu}$, bien que le matériel ait toujours été (et continue d'être) à disposition des chercheurs de Nankin et de Shanghai. Au Conservatoire de musique de Shanghai, où je travaillais pendant les intervalles entre mes voyages d'enquête, il était difficile de trouver des enregistrements historiques de chants populaires de la région de Wu. Le conservatoire a une discothèque, mais le public n'a pas accès à tous les enregistrements, et il n'existe pas de catalogue des enregistrements faits sur le terrain par les étudiants et les professeurs de l'institut. La plupart des bons enregistrements sont la propriété privée des professeurs. Durant ces vingt-cinq dernières années, le matériel enregistré a été très mal entretenu et en grande partie détruit. Il est tout à fait compréhensible que beaucoup de gens ne désirent pas mettre leur propre matériel à la disposition des collègues. Cependant, 
quelques professeurs et étudiants me donnèrent des bandes intéressantes de chants $\mathrm{Wu}$, enregistrées dans les années 1950, qui m’ont au moins permis de comparer les différentes versions d'une chanson sur une période de plus de trente ans.

\section{En guise de conclusion}

J'espère que mon travail dans la région de Wu incitera d'autres personnes à se consacrer à la recherche et à l'étude des chants populaires chinois. Les chants et les voix des chanteurs du Jiangsu méritent d'être conservés. L'idéal serait de les sauvegarder sur bandes magnétiques et sur disques pour le bénéfice des générations futures. Il est évident que l'étiolement de la pratique du chant dans la région de Wu reflète, voire anticipe, le sort de nombreuses cultures traditionnelles. Aucune culture ne peut durer éternellement. Notre but n'est pas de momifier celle du chant populaire $\mathrm{Wu}$, mais de développer une meilleure compréhension de cette tradition et peut-être d'y trouver de nouvelles significations à la lumière de la société humaine en mutation. En outre, la recherche musicale sur le terrain est une merveilleuse aventure. Sonder l'esprit des Chinois, savourer de nouveaux plats et de nouveaux airs, rire ensemble... Peut on imaginer une meilleure approche d'un pays et de son peuple? Certainement, si vous souhaitez voir de la Chine plus que ce qui tombe sous vos yeux...

\section{BIBLIOGRAPHIE}

Anon. 1, 1989, Jiangsusheng shi xian jingji. [Economie des cantons et comtés de la Province du Jiangsu]. Jiangsusheng tongiieju ed. Nanjing : Zhongguo tongji chubanshe.

Anon. 2, 1981, Zhejiangsheng dituce. [Atlas de la Province de Zhejiang]. Zhejiangsheng cehuiju ed. Shanghai : Ditu chubanshe.

BAYARD Samuel Preston, 1939, « Aspects of Melodic Kinship and Variation in British-American Folk-tunes ». Papers of the American Musicology Society, New York : 122ff.

BRAILOIU Constantin, 1984a, Problems of Ethnomusicology. Articles de Brăiloiu éd. et trad. par A.L. Llyod, Cambridge (UK) : Cambridge Univ. Press.

BRAILOIU Constantin, 1984b, Musical Folklore. In Brăiloiu 1984a : 1-58. (à l'origine en français dans Musica aeterna. $2^{\mathrm{e}}$ éd. Zürich : M.S. Metz, 1949).

BRAILOIU Constantin, 1984c, « Outline of a method of musical folklore ». In Brăiloiu 1984a : 59-85. (à l'origine en roumain dans Boabe de griu II/4, Bucarest : Editura muzicala, 1931).

BURKE Peter, 1994, Popular Culture in Early Modern Europe. Première édition par Maurice Temple Smith Ltd, London, 1978. Réédition revue par Scholar Press, Hants / Cambridge Univ. Press.

DONG Sen et al. (éds.), 1984, Wuge [Chants Wu] des séries Zhongguo geyao congshu [Séries de chants populaires chinois]. Beijing : Zhongguo minjian wenyi chubanshe. 
FEI Hsiao Tung et al., 1986, Small Towns in China - Functions, Problems \& Prospects. Beijing : New World Press.

FINNEGAN Ruth, 1988, Literacy and Orality, Studies in the Technology of Communication. Oxford : Basil Blackwell.

FOLEY John Miles, 1988, The Theory of Oral Composition. Bloomington : Indiana UP.

GU Jiegang, 1936, «Wuge xiaoshi » [Brève histoire des chants Wu]. Geyao [Chants populaires]

2/23:1-8.

JIANG Bin (ed.), 1989, Jiangnan shi da minjian xushishi. changpian wugeji Wuge ji [Dix longs poèmes narratifs du Sud du Jiansu. Une collection de longs chants Wu]. Shanghai : Shanghai wenyi chubanshe.

JIN Sha et al. (eds.), 1984, Suzhou minjian gequ [Chants populaires de Suzhou. 2 tomes]. vol. 5 \& 6 dans la série Suzhou minzu minjian yinyue jicheng [Anthologie de la musique populaire de Suzhou. vol. 1-8]. Suzhou : Suzhoushi wenlian. Polycopié.

JIN Tianlin et al. (eds.), 1987, Jianshan min'ge [Chants populaires du Jianshan]. Publ. par le wenhuaju , wenlian et whenhuaguan (bureaux de la culture) de Jianshanxian. Province du Zhejiang.

LORD Albert Bates, 1976, The Singer of Tales. Première édition par Harvard Univ. Press, Cambridge Mass. en 1960. Nouvelle édition : Atheneum.

MA Hanmin et al. (eds), 1979, Wuge xinji [Nouvelle collection de chants Wu]. Suzhou : Suzhoushi wenlian.

SCHIMMELPENNINCK Antoinet, 1995, «Field Report from the Yangzi Delta : Chinese Folk Singers in Jiangsu Province (1) ». Chime. No $8: 32-58$.

SCHIMMELPENNINCK Antoinet, 1996, «Field Report from the Yangzi Delta : Chinese Folk Singers in Jiangsu Province (2) ». Chime. No 9 (à paraître en 1996).

SHI Lin et al.(eds.), 1982, Zhongguo Minjian gequ jicheng, Jiangsu juan 1-5 [Anthologie de chants populaires chinois. Jiangsu, tomes 1-5]. Polycopié, Nanjing.

TREITLER Leo, 1974, « Homer and Gregory : the transmission of epic poetry and plainchant ». The Musical Quarterly 60/3 : 333-372.

XU Wenchu (ed.), 1988, Zhongguo minjian wenxue jicheng. Wujiangxian ziliaoben [Anthologie de la littérature populaire chinoise]. Recueil de matériaux provenant de la municipalité de Wujiang). Wujiangxian minjian wengue jicheng bangongshi.

ZHU Hairong (ed.), 1987, Zhongguo minjian wenxue jicheng. Wuxixian changpian xushi Wuge ji [Anthologie de la littérature populaire chinoise. Une collection de longs chants narratifs Wu de la municipalité de Wuxi]. Wuxi : Jiangsu chuban zongshe.

\section{NOTES}

1. Traduit de l'anglais par Ramèche Goharian.

2. Cet article est la version abrégée d'un chapitre de ma thèse de doctorat (en cours de préparation) sur la musique et la langue du Shan'ge dans le sud du Jiangsu. Cette étude a été rendue possible grâce aux bourses octroyées par l'Ecole de Recherche CNWS (Université de Leiden), l'Organisation néerlandaise pour la recherche scientifique NWO et la Chiang Ching-kuo Foundation for International Scholarly Exchange (Taiwan). Je suis redevable à Frank 
Kouwenhoven de m'avoir assistée dans toutes les étapes de ma recherche, ainsi qu'à Willeke Van de Ree de m'avoir aidée à lire la traduction française de cet article.

3. Gu Jiegang (1936:2/23) a cherché les traces du nom Wu dans l'histoire.

4. Extrapolé à partir des tableaux de répartition de la population dans Jiangsusheng shi xian jingji ( 1989 :64-66) et Zhejiangsheng dituce (1981:14).

5. La Société pour la recherche sur le chant populaire $\mathrm{Wu}$ (Wuge xuehui) réunit environ deux cents membres, chanteurs et collecteurs qui s'intéressent à la tradition locale du chant, plus particulièrement aux textes des chansons et dans une moindre mesure à leur musique. La Société publie des chants et contribue à plusieurs revues de littérature populaire publiées à Shanghai ou ailleurs. Son centre d'activité actuel est le musée du folklore de Suzhou (Suzhou minsu bowuguan).

6. Pour plus d'informations détaillées sur les chanteurs et leurs milieux, voir mes articles à paraitre dans Chime, vol 8 et 9 (publication en 1995 \& 1996).

7. A noter que les périodes exactes du nongmang peuvent varier d'une région à l'autre.

8. Dans les troisième et quatrième années de mon travail sur le terrain, je découvris de petites communautés de chanteurs à Luxu, Dingzha et Taozhang, mais à ce stade, il n'était pas pratique de se lancer dans des études villageoises détaillées.

9. Cf. la préface d'Alan Dundes à John Miles Foley (1988:ix-xii), et diverses sections du livre de Foley, surtout le chapitre IV, pp. 57-93.

10. Publié pour la première fois par Temple Smith, London, 1978.

11. Cf. Fei Hsiao Tung (1986:60).

12. Surtout deux volumes généraux : Dong Sen ed.(1984) et Ma Hanmin et al eds.(1979) ainsi qu'un volume spécial de poésie épique populaire, Jiang Bin ed.(1989).

13. Zhu Hairong ed.(1987) ; Xu Wenchu ed.(1988) et Jin Tianlin et al eds.(1987).

14. Shi Lin et al ed.(1982). Jin Sha et al eds.(1984).

\section{RÉSUMÉS}

Dans cet article, l'auteur présente ses méthodes de travail sur le terrain et les problèmes qu'elle a rencontrés pendant ses recherches sur la tradition du chant populaire dans la province chinoise du Jiangsu Sud. Dans un premier temps, elle situe brièvement la région dialectale de Wu où elle a mené l'essentiel de ses recherches et décrit la tradition du chant populaire telle qu'elle se présente aujourd'hui dans le Jiangsu. Puis elle expose en détail son travail effectif sur le terrain et traite des problèmes du transport et de la communication, de l'organisation des séances d'enregistrement, de la collecte et du traitement informatique des données et, bien entendu, de la quête des chanteurs populaires.

This is a survey of fieldwork methods and the problems with which the author was confronted during research into the folk song culture of Southern Jiangsu, China. This article starts with a brief description of the Wu dialect area, where most of the research was carried out, and a description of the folk song culture as it can be found in Jiangsu today. This is followed by a detailed account of the actual fieldwork, looking at such aspects as transport, communication, the organisation of recording sessions, the collecting and processing of data and such basic questions as how to find folk singers in the first place. 


\section{AUTEURS}

\section{ANTOINET SCHIMMELPENNINCK}

Antoinet Schimmelpenninck, née en 1962, a obtenu son diplôme de sinologie en 1988. Elle a commencé ses recherches sur le chant populaire chinois en 1986 et a été acceptée comme doctorante à l'Ecole de Recherche CNWS de l'université de Leiden (Hollande). Avec son mari, Frank Kouwenhoven, elle a mené d'importantes recherches sur le terrain dans les provinces de Zhejiang et du Jiangsu. Elle termine en ce moment sa thèse de doctorat sur le chant populaire chinois. En 1990, elle a participé à la création de la Fondation européenne pour la recherche sur la musique chinoise (CHIME) et elle est co-éditrice de Chime Journal. 